\title{
ИНФОРМАЦИОННЫЙ КАПИТАЛ ПРЕДПРИЯТИЯ КАК ЦЕЛЕВОЙ ПОКАЗАТЕЛЬ РАЗВИТИЯ В РАМКАХ ЦИФРОВЫХ ЭКОНОМИЧЕСКИХ СИСТЕМ
}

\author{
(c) 2020 Родионов Дмитрий Григорьевич \\ доктор экономических наук, профессор \\ Высшая инженерно-экономическая школа \\ Санкт-Петербургский политехнический университет Петра Великого (СПбПУ), \\ Россия, Санкт-Петербург \\ E-mail: dmitry.rodionov@spbstu.ru \\ (c) 2020 Конников Евгений Александрович \\ кандидат экономических наук, Высшая инженерно-экономическая школа \\ Санкт-Петербургский политехнический университет Петра Великого (СПбПУ), \\ Россия, Санкт-Петербург \\ E-mail: konnikov.evgeniy@gmail.com \\ (c) 2020 Алферьев Дмитрий Александрович \\ Высшая инженерно-экономическая школа \\ Санкт-Петербургский политехнический университет Петра Великого (СПбПУ), \\ Россия, Санкт-Петербург \\ E-mail: alferev_1991@mail.ru
}

Процесс цифровизации экономики является крайне комплексным, и под его воздействием трансформируются многие паттерны взаимодействия экономических субъектов. По результатам последовательного формирования цифровой информационной среды, характеризующейся высокой скоростью и широтой распространения информации, процесс потребления значительно трансформировался, и на данный момент цифровая информационная среда фактически выступает надстройкой существующих экономических систем, в рамках которой формируется комплексная потребительская информация. В следствии описанного процесса, на данный момент сформировались механизмы обратного воздействия, в соответствии с которыми вещественная реальность трансформируется под влияние процессов, зародившихся в цифровой информационной среде. Таким образом, в рамках конкурентного взаимодействия все большее влияние приобретает специфика восприятия предприятия в цифровой информационной среде со стороны ее субъектов. Количественное выражение данного восприятия может быть названо информационным капиталом. В рамках данной статьи авторы разрабатывают инструмент оценки данного параметра, а также описывают механизмы его формирования и изменения во времени.

Ключевые слова: информационный капитал, цифровизация, цифровая экономическая система, тональность, устойчивость, информационная среда.

Процесс цифровизации экономических систем неизменно способствует развитию цифровой экономической надстройки, которая также может быть названа информационной средой. На базовом уровне развития цифровая среда исключительно отражала состояние вещественной реальности и способствовала распространению объективной экономической информации. На данный момент цифровая среда содержит не только механизмы обмена, но и механизмы генерации информации, а ее свойства, в частно- сти скорость и широта распространения информации, привели к масштабной трансформации паттернов потребительского поведения, что в конечном итоге привело к формированию механизмов обратного воздействия, в соответствии с которыми цифровая среда может трансформировать вещественную реальность. В следствии данного процесс, у предприятий сформировалось новое свойство, которое может быть названо информационным капиталом. Под информационным капиталом предприятия стоит 
понимать относительную величину, характеризующую восприятие предприятия субъектами информационной среды. Данное восприятие является многомерным, в рамках рыночных связей оно количественно может выражаться в характеристиках спроса на товары и услуги предприятия и товары и услуги конкурентов предприятия, в рамках связей между бизнеспартнерами и инвесторами оно может выражаться как в условиях инвестирования, так и в горизонте планирования, а в рамках связей между предприятием и государством данное восприятие может выражаться в частоте налоговых и иных проверок. Надо акцентировать внимание на том, что данная величина является относительной и, как следствие, должна измеряться посредствам ранговой шкалы. Сущностно, данная величина отражает силу и вектор воздействия информационной среды на связи между предприятием и субъектами информационной среды, среди которых в первую очередь можно выделить потребителей, которых в свою очередь можно разделить на фактических и потенциальных потребителей, предприятия - конкуренты, предприятия - комплименторы, агенты информационной среды (СМИ, блогеры и иные лидеры мнений) и представители государственного аппарата (налоговые органы, таможенные органы и т.д.). Для целей оценки данного параметра может быть предложена специализированная математическая модель, позволяющая сравнить данный ранговый параметр с соответствующими параметрами конкурентов. Дня целей формирования данной модели наиболее целесообразно использовать нечетко-множественный инструментарий моделирования. Данный параметр может быть назван «Индекс информационного капитала», а первичным этапом разработки модели оценки является идентификация факторов, оказывающих влияние на исследуемый комплексный показатель. Индекс информационного капитала предлагается дифференцировать на три базовые компоненты:

1. Индекс направленного восприятия предприятия $\left(\mathrm{I}_{d}\right)$. Данный индекс отражает общее усредненное восприятие предприятия со стороны субъектов информационной среды. Несмотря на общую абстрактность, именно данный индекс отражает воздействие самого предприятия на информационную среду. Колебания данного индекса являются следствием разнонаправленной деятельности самого предприятия и качества представления данной деятельности в цифровой среде. Данный индекс определяется экспертно и измеряется в условных баллах.

2. Индекс тональности информационной среды $\left(\mathrm{I}_{\mathrm{t}}\right)$. Данный индекс отражает тональный окрас информационного фона. Механизм влияния данного показателя информационный капитал предприятия можно описать посредствам трансформации потребительского поведения вследствие изменения тональности информационной среды. В качестве базового индикатора трансформации потребительского поведения может выступать уровень инфляции. В мировой науке выделяется множество возможных причин роста инфляции, однако ключевой является увеличение совокупного спроса. По нашему мнению, совокупный спрос в условиях устойчивой экономики может колебаться вследствие изменения инфляционных ожиданий и вследствие изменения тональности информационной среды. Инфляционные ожидания являются следствием наблюдения инфляционных колебаний прошлых периодов, следовательно их можно выразить в виде авторегрессионной переменной. Тональность информационной среды можно выразить в виде множества показателей. В данном случае для оценки тональности информационной среды использовалась открытая библиотека для семантического анализа средствами языка python - «Dostoevsky». Данная библиотека была сформирована на основе базы данных «RuSentiment dataset» и ее точность в соответствии с критерием F1 составляет 0,71, что является необходимым и достаточным уровнем точности. Аналитический массив формировался на основе новостных заголовков и аннотаций новостей, источниками которых послужили: «МК: Новости России и мира», «РИА Новости» и «Яндекс.Новости». Выбор обусловлен тем, что данные новостные порталы являются наиболее популярными в российском сегменте интернета, о чем свидетельствует рейтинг посещаемости, а также они обладают универсальной направленностью новостного контента. Процедура парсинга информации и формирования датафрейма подразумевает сбор ежедневного массива новостных заголовков и аннотаций новостей со всех трех ресурсов, на промежутке с 01.01.2016 года по 01.02.2020 года, и последующее агрегирование данных в ежемесячные массивы, по результатам чего было сформирован массив, состоящий из 49 наблюдений. Библиотека 
Dostoevsky позволяет получить по результатам анализа тональности Negative и Positive характеристику текстового массива, соотношение которых позволяет образовать множество возможных регрессоров. По результатам анализа была получена следующая регрессионная модель:

$$
0,89+0,95 * I_{n-1}-0,09 *\left(\frac{\text { Negative }_{n-1}}{\text { Positive }_{n-1}}\right)^{2}
$$

Где:

$\mathrm{I}_{\mathrm{n}}$ - уровень инфляции за месяц $\mathrm{n}$;

$\mathrm{I}_{\mathrm{n}-1}$ - уровень инфляции за месяц n-1 (авторегрессионная компонента, отражающая уровень инфляционных ожиданий);

Negative $_{\mathrm{n}-1}-$ уровень негативной тональности информационной среды за месяц n-1;

Positive $_{\mathrm{n}-1}-$ уровень позитивной тональности информационной среды за месяц n-1.

Нормированный $\mathrm{R}^{2}$ данного уравнения составил 0,96, что говорит о крайне высоком качестве модели. Стандартная ошибка составляет около 6\% a p-level каждого из регрессоров не превышает 0,05. Форма регрессора, отражающего влияние тональности информационной среды определена эмпирически (критерием отбора выступал уменьшающийся p-level), и в соответствии с ней можно утверждать, что тональность информационной среды, в контексте влияния на информационный капитал предприятия, определяется квадратом отношения негативной и позитивной тональной составляющей. При этом, влияние данного индекса на уровень инфляции носит отрицательный характер. Данный факт полностью подтверждает гипотезу, согласно которой при увеличении негативной тональной составляющей человек склонен снижать уровень своего потребления и наращивать сбережения. В свою очередь, влияние данного индекса на индекс информационного капитала можно рассмотреть, как обратная величина от данного индекса. Следовательно индекс тональности информационной среды рассчитывается следующим образом:

$$
I_{t}=-\left(\frac{\text { Negative }}{\text { Positive }}\right)^{2}
$$

3. Индекс устойчивости информационной среды $\left(\mathrm{I}_{\mathrm{s}}\right)$. Данный индекс отражает волатильность содержательной составляющей информа- ционной среды. При высоком уровне данного показателя информационная среда может характеризоваться как нестабильная. Содержательная составляющая информационной среды может характеризоваться облаком токенов и частотой упоминания того или иного токена. Появление новых токенов может говорить о трансформации содержательной составляющей информационной среды, а их доля в свою очередь отражает ее волатильность. Данный показатель может быть определен следующим обра3ом:

$$
V=\frac{\sum_{1}^{i} n e w \cdot F_{t_{n}}}{\sum_{1}^{j} F_{t_{n-1}}}
$$

Где:

V - показатель трансформации содержательной составляющей информационной среды; $n-1$;

$\mathrm{F}_{\mathrm{t}_{\mathrm{n}-1}}$ - частота появления токена в периоде

new. $\mathrm{F}_{\mathrm{t}_{\mathrm{n}}}$ - частота появления нового токена в периоде $\mathrm{n}$;

i - число новых токенов периоде n;

$\mathrm{j}$ - общее число токенов периоде n-1.

В качестве токенов предлагается использовать лемматизирвоанные словоформы, присутствующие в информационной среде. Для данных целей может эффективно использоваться открытая библиотека на языке python - «NLTK».

В соответствии с приведенными тезисами, данные факторы в полной мере характеризуют влияние информационной среды на информационный капитал предприятия, и формируют нечетко-множественную модель оценки данной величины. В рамках данной модели выделена одна интегральная лингвистическая переменная - «Индекса информационного капитала (Е.С.I)». Базовое терм множество в случае данного индекса было решено не дифференцировать на подмножества. Однако, данный интегральный показатель является результирующим показателем модели. Для каждого частного показателя также сформирована лингвистическая переменная - «Значение частного показателя». Базовое терм множество в случае данных показателей имеет по 5 подмножества:

1. Недопустимое значение показателя;

2. Низкое значение показателя; 
3. Среднее значение показателя;

4. Высокое значение показателя;

5. Показательное значение показателя.

В качестве классификатора для частных показателей выбран стандартный пятиуровневый 01-классификатор. В классификаторе в качестве носителя лингвистической переменной выступает отрезок вещественной оси $[0 ; 1]$ (01-носитель). Для описания вида подмножеств терм-множества введена система из пяти функций принадлежности, характеризующих степень принадлежности отрезка значений 01-носителя заданному подмножеству (таблица 1).

Распределение удельного веса влияния используемых показателей исключительно неравномерно. Данный факт обусловлен тем, что индекс направленного восприятия предприятия оказывает значительно большее влияние на интегральный показатель. В тоже время удельный вес влияния иных показателей сопоставим, в следствии чего распределение имеет следующий вид: $r\left\{\mathrm{I}_{\mathrm{d}} ; \mathrm{I}_{\mathrm{t}} ; \mathrm{I}_{\mathrm{s}}\right\}=\{0.6 ; 0.2 ; 0.2\}$.

Приведённые классификаторы могут быть представлены в виде трапециевидных функций принадлежности, где по оси ординат обозначены значения функций принадлежности (от 0 до 1), a по оси абсцисс - термы. При этом верхнее основание трапеции соответствует абсолютной уверенности эксперта в надежность классификации, а нижнее основание характеризует уверенность в том, что иные значения интервала $(0 ; 1)$ не попадают в выбранное нечеткое подмножество. Боковые грани трапеций отражают колебание суждения эксперта о принадлежности конкретного отрезка на 01-носителе к тому или иному терму. Существует пять узловых точек: $\{0,1 ; 0,3$; $0,5 ; 0,7 ; 0,9\}$. По результатам расчета каждого из частных показателей, проводится распознавание их значений по критерию $\lambda_{\mathrm{ij}} \in[0 ; 1]$. Данный показатель соотносит значения частных показа- телей со значениями 01-носителя:

$$
\lambda_{i J}=1-\frac{X_{i}-a_{3}^{*}}{a_{4}^{*}-a_{3}^{*}}
$$

Где $\mathrm{a}_{3}^{*}$ и $\mathrm{a}_{4}{ }^{*}-$ Т-числа і-го подмножества терм-множества.

По результатам распознавания значений частных показателей, рассчитываются интегральные показатели:

E. C. I $=\sum_{1}^{3} p_{j} \times r_{i} \times \lambda_{i J}$

Где $\mathrm{p}_{\mathrm{j}}$ - узловые точки 01-носителя:

$$
p_{j}=0,9-0,2 \times(j-1)
$$

Где $\mathrm{j}$ - номер подмножеств базового терм множества

Приведенная нечетко-множественная модель, позволяет оценить индекса информационного капитала в диапазоне от 0 до 1. Однако, данная модель исследует исключительно изолированное влияние данных показателей на информационный капитал предприятия. При этом, они могут оказывать влияние друг на друга. В условиях стабильной информационной среды вне контекста направленных изменений, взаимодействие данных показателей можно рассмотреть, как замкнутую систему. Изменение каждого из представленных параметров в первую очередь имеет авторегрессионный характер. Следовательно, в первую очередь изменение каждого из них будет зависеть от его прошлого значения, скорректированного на величину чувствительности соответствующего параметра к изменениям. На изменение направленного восприятия предприятия приращение иных параметров оказывает положительный эффект.

Таблица 1. Т-числа $\{\mathbf{y}\}$ для значений лингвистической переменной

\begin{tabular}{|l|c|c|c|}
\hline \multicolumn{1}{|c|}{ Подмножество } & \multicolumn{3}{|c|}{ Показатель } \\
\cline { 2 - 4 } & $\mathbf{I}_{\mathbf{d}}$ & $\mathbf{I}_{\mathbf{t}}$ & $\mathbf{I}_{\mathbf{S}}$ \\
\hline Недопустимое значение показателя & $(1 ; 1 ; 2 ; 3)$ & $(-81 ;-81 ;-70.9 ;-60.8)$ & $(0 ; 0 ; 0.1 ; 0.2)$ \\
\hline Низкое значение показателя & $(2 ; 3 ; 4 ; 5)$ & $(-70.9 ;-60.8 ;-50.6 ;-40.5)$ & $(0.1 ; 0.2 ; 0.3 ; 0.4)$ \\
\hline Среднее значение показателя & $(4 ; 5 ; 6 ; 7)$ & $(-50.6 ;-40.5 ;-30.4 ;-20.6)$ & $(0.3 ; 0.4 ; 0.5 ; 0.6)$ \\
\hline Высокое значение показателя & $(6 ; 7 ; 8 ; 10)$ & $(-30.4 ;-20.6 ;-10.1 ;-0.01)$ & $(0.5 ; 0.6 ; 0.7 ; 0.8)$ \\
\hline Показательное значение показателя & $(8 ; 10 ; 10 ; 10)$ & $(-10.1 ;-0.01 ;-0.01 ;-0.01)$ & $(0.7 ; 0.8 ; 0.8 ; 0.8)$ \\
\hline
\end{tabular}


Однако, их кумулятивное влияние должно быть скорректировано с учетом установленного ранее удельного веса. Тональность информационной среды также приращается за счет приращения остальных параметров, однако корректировка удельного веса влияния в данном случае уже не требуется. Устойчивость информационной среды, наоборот, уменьшается при приращение остальных показателей. Таким образом, взаимовлияние данных показателей (в стандартизированной форме) можно представить в виде следующей системы дифференциальных уравнений:

$$
\left\{\begin{array}{l}
\frac{d I_{s_{t}}}{d t}=\alpha * I_{s}-I_{t} * I_{d} \\
\frac{d I_{t_{t}}}{d t}=\beta * I_{t}+I_{s} * I_{d} \\
\frac{d I_{d_{t}}}{d t}=d * I_{d}+\frac{I_{s} * I_{t}}{3}
\end{array}\right.
$$

\section{Где:}

$\alpha$ - коэффициент чувствительности индекса устойчивости информационной среды к его изменениям в прошлом периоде;

$\beta$ - коэффициент чувствительности индекса тональности информационной среды к его изменениям в прошлом периоде;

$d$ - коэффициент чувствительности индекса направленного восприятия предприятия к его изменениям в прошлом периоде.

Как можно видеть, представленная система дифференциальный уравнений имеет вид странного аттрактора Чена-Ли (The Chen-Lee Attractor), что позволяет сформировать множество неустойчивых траекторий, обладающих хаотическим характером. Данной системе свойственен динамический хаос. Приведем пример для линейного изменения значений t от 0 до 100 , образующего 40000 наблюдений. При базовых значениях показателей $\mathrm{I}_{\mathrm{d}}, \mathrm{I}_{\mathrm{t}}$ и $\mathrm{I}_{\mathrm{s}}$ соответственно равными 1, 1 и 1 и базовых значениях коэффициентов $\alpha, \beta$ и $d$ составляющих $5,-10$ и -0.38 , данный аттрактор примет вид, показанный на рис. 1.

В соответствии с полученным данными можно утверждаться, что тональность и устойчивость информационной среды имеют неоднородную динамику. Они гармонически колеблются при изменении направленного восприятия предприятия. При этом наблюдается область относительного покоя, которая характеризует

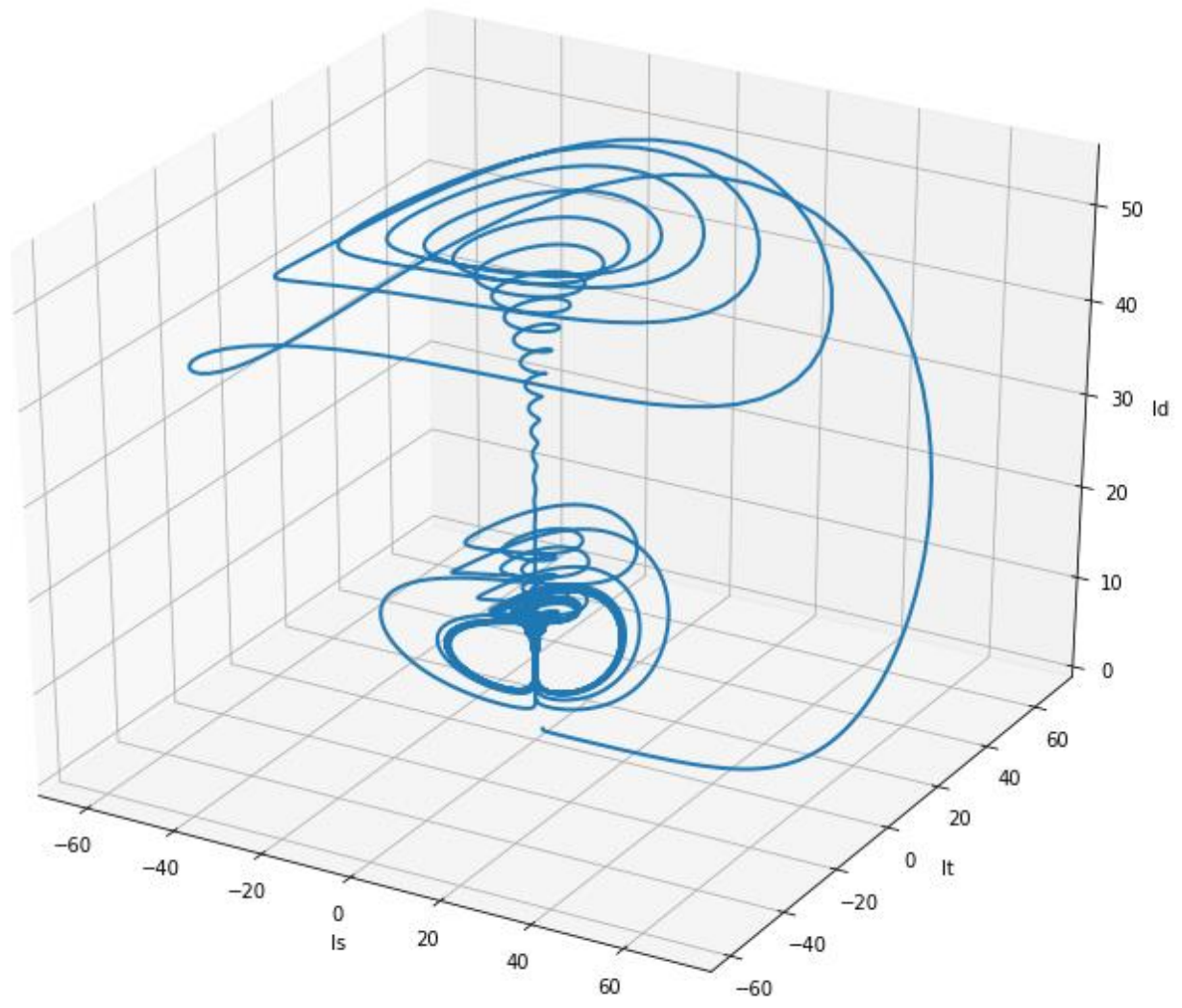

Рисунок 1. Изменение целевых параметров системы при базовых значениях параметров аттрактора Чена-Ли 
стабильное приращение положительного информационного потока, относительно предприятия. Отклонения приращения данного потока провоцируют изменения и как следствие хаотические колебания тональности и устойчивости информационной среды. Следовательно, можно заключить, что значительное приращение направленного информационного потока со стороны предприятия, или наоборот его резкое снижение, могут провоцировать хаотические изменения в информационной среде, и, как следствие, приводить к непрогнозируемым результатам.

В соответствии с полученными результатами, можно утверждать, что информационный капитал предприятия является многомерной частично-управляемой категорией. Приведенные в научном сообществе тезисы относительно связи информационного капитала и его деловой репутации верны лишь частично, в первую очередь, потому что деловая репутация формируется исключительно как следствие действий предприятия. Волатильность информационного капитала обеспечивается в равной степени как действиями самого предприятия, так и состоянием информационной среды. На рисунке 2 приведен процесс формирования информационного капитала.

Как можно видеть на рисунке, информационный капитал формируется вне предприятия, под воздействием информационной среды. Предприятие формирует информационный поток, который поступает в информационную среду. Данный поток содержит как формальную информацию (статистику продаж, миссию компании, пресс-релизы руководства компании, данный бухгалтерского учета и т.д.), так и неформальную информацию (отзывы сотрудников и руководителей, страницы сотрудников предприятия в социальных сетях и т.д.). Данный совокупный информационный поток достаточно неоднородный и дифференцированный в зависимости от принимающей аудитории. Помимо информационного потока, предприятие также направляет в информационную среду поток своих товаров, работ и услуг (ТРУ), на основе которых формируется основное восприятие предприятия со стороны фактических потребителей. Также предприятие формирует исходящий поток физического воздействия. Данное воздействие можно разделить на социальное, экологическое, политическое и научно-технологическое. Содержание данных потоков, попадая в информационную среду начинает активно взаимодействовать с содержанием потоков иных предприятий, а также со всей совокупностью субъектов информационной среды. Именно данное взаимодействие и формирует информационный капитал предприятия. Исходящие от предприятия потоки могут быть синхронизированы с информационной средой, что в свою очередь позволит обеспечить относительную стабильность информационного капитала.

Информационная среда предприятия

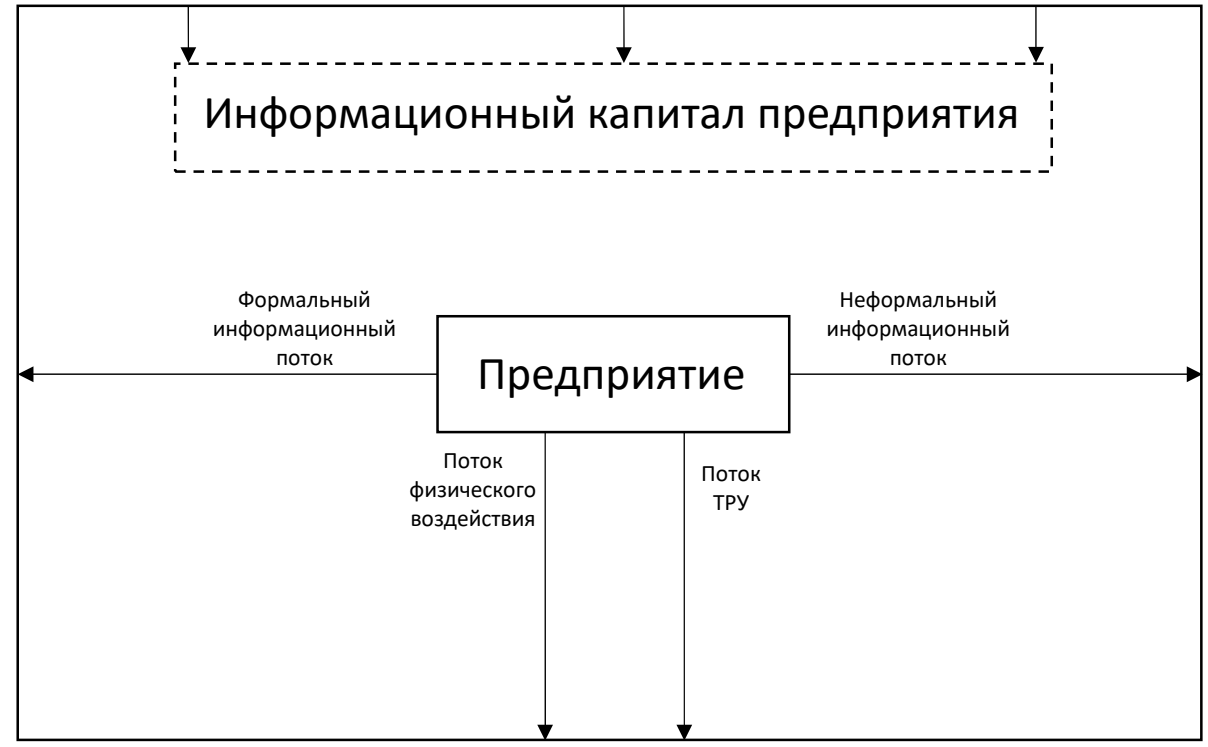

Рисунок 2. Процесс формирования информационного капитала предприятия 


\section{Библиографический список}

1. Багоян Е.Г., Демьянец М. В., Десятниченко Д. Ю. и др. Основы цифровой экономики // учебник для и практикум для вузов / Москва, 2020. Сер. Высшее образование

2. Конникова О.А., Конников Е.А. Синтаксический анализ поведения потребителей в цифровой среде // В книге: Маркетинг сотворчества и глобальные коммуникации доверия. Багиев Г. Л., Юлдашева О. У., Шубаева В. Г. и др. Посвящается 90-летию СПбГЭУ и 30-летию кафедры маркетинга. Санкт-Петербург, 2020. С. $132-145$.

3. Родионов Д.Г., Ялымов С. В., Конников Е. А. Влияние информационной среды на субъекты малого и среднего предпринимательства // Экономические науки. 2020. № 189. С. 86-91.

4. Фриц В., Натараян Р., Аренков И. А. и др. Инновации маркетинговых моделей российских компаний в эпоху диджитал трансформации // Коллективная монография / Санкт-Петербург, 2018. 